\title{
Astrophysics \& Space Science subscribes to the Singapore Statement on Research Integrity
}

\author{
Michael Dopita
}

Received: 29 November 2011 / Accepted: 29 November 2011 / Published online: 5 January 2012

(C) Springer Science+Business Media B.V. 2011

The Singapore Statement on Research Integrity was developed as part of the 2nd World Conference on Research Integrity, held in Singapore 21-24 July 2010. This provides a global guide to the responsible conduct of research, and is available at: www.singaporestatement.org.

The Editors of Astrophysics \& Space Science wish to announce that they, and the Journal, subscribe to, and will be bound by, the principles espoused in the Singapore Statement. Specifically:

- Honesty in all aspects of research

- Accountability in the conduct of research

- Professional courtesy and fairness in working with others

- Good stewardship of research on behalf of others

In respect to the responsibilities this implies for our authors these are as follows:

- Researchers should employ appropriate research methods, base conclusions on critical analysis of the evidence and report findings and interpretations fully and objectively.

- Researchers should take responsibility for their contributions to all publications, funding applications, reports and other representations of their research. Lists of authors should include all those and only those who meet applicable authorship criteria.

M. Dopita $(\bowtie)$

Mount Stromlo and Siding Spring Observatories, Research School of Astronomy and Astrophysics, Australian National University, Cotter Road, Weston Creek, ACT 2611, Australia e-mail: michael.dopita@anu.edu.au

M. Dopita

Astronomy Department, King Abdulaziz University,

P.O. Box 80203, Jeddah, Saudi Arabia
- Researchers should acknowledge in publications the names and roles of those who made significant contributions to the research, including writers, funders, sponsors, and others, but do not meet authorship criteria.

Insofar as our referees are concerned, they are requested to provide fair, prompt and rigorous evaluations and required to respect confidentiality when reviewing the work of others.

Readers or reviewers should report to the Editors any suspected research misconduct, including fabrication, falsification or plagiarism, and other irresponsible research practices that undermine the trustworthiness of research, such as carelessness, improperly listing authors, failing to report conflicting data, or the use of misleading analytical methods.

For their part the Editors will endeavor to provide authors with referee reports and decisions on papers in the shortest practicable timeframe, and to provide assistance to authors encountering difficulties in submission. The Editors also undertake to fully investigate and to appropriately respond to any allegations of misconduct and to withhold the identity of those making the allegations from the party concerned, except where permission to do so is explicitly granted by the complainant. When misconduct or other irresponsible research practice is confirmed, appropriate actions will be taken, including correcting the research record.

Michael Dopita, Editor in Chief 